\title{
Impunidad de personas con patrones culturales distintos ${ }^{1}$
}

José HurTado Pozo

\section{Introducción}

De manera interesante, Luis Francia ${ }^{2}$ aborda el complejo problema que representa la diversidad cultural de nuestra sociedad para el derecho penal. El esfuerzo hecho por el autor merece bien un comentario, un poco más extenso de lo acostumbrado, de parte de un viejo aficionado al derecho penal. El propósito es alentar el diálogo crítico y serio, que falta entre nosotros con frecuencia.

El título del presente comentario es una afirmación hecha por Francia de manera absoluta y reiterativa. Así, sostiene que «en ningún caso el derecho penal oficial penará a personas que hayan cometido un hecho delictivo en razón de un patrón general de valores culturales distintos a los del derecho penal oficial» (p. 511 s.) y, más brevemente, "el derecho penal oficial no tiene razón para penar a individuos con patrones culturales distintos" (p. 512).

Para llegar a esta conclusión, Francia parte, primero, de una serie de afirmaciones esquemáticas sobre el sistema penal oficial. Siguiendo los postulados de la criminología crítica, señala que el derecho penal legitima el sistema global; que, en la práctica, el derecho penal criminaliza de manera selectiva a los miembros de los sectores populares; que el derecho penal, en lugar de resolver conflictos, origina conflictos y problemas mayores; $y$, por último, que no brinda seguridad a la población, en contra de lo afirmado oficialmente.

Dedicamos este modesto trabajo a Richi Villanueva, en agradecimiento a las horas que pasamos juntos discutiendo los aspectos centrales de la problemática apenas presentada y discutida en esta ocasión. Errores e imprecisiones son de nuestra sola responsabilidad.

2 Luis Francia, "Pluralidad cultural y derecho penal", en Derecho, PUCP, n. 47 , Lima, 1993 , pp. 493 y ss. 
Después de ubicar en este contexto el problema de la pluralidad cultural, Francia presenta y critica la manera como legislativamente se ha afrontado este problema. Así, critica el etnocentrismo con que se clasificó a los peruanos, en el Código Penal de 1924, en civilizados, indígenas semicivilizados y salvajes. Lo mismo hace con el tratamiento que se da a los dos últimos grupos, como incapaces o inmaduros. A todos ellos los considera normales y, en consecuencia, rechaza que su diversidad cultural sea regulada como si fuera un problema de incapacidad penal. Haciendo lo contrario, los redactores del Código de 1924 proclamaron falsamente, según Francia, la «superioridad de la sociedad occidental sobre las demás, y también falsamente la superioridad del hombre occidental». De esta manera, llega a afirmar, primero, que el código derogado escondía "una política etnocida y violatoria del derecho a la autonomía cultural, a la diferencia e identidad cultural", y, segundo, que "era una manera de legitimar un modelo autoritario y antidemocrático" (p. 500).

Respecto al Código de 1991, Francia admite que lo dispuesto en el artículo 15 constituye un avance en la medida que reconoce la heterogeneidad cultural de nuestra sociedad y regula esta situación como un aspecto de culpabilidad. En su opinión, la solución es incompleta: no basta que se reconozcan los «diversos mecanismos de regulación social y resolución de conflictos no oficiales", ya que es indispensable que se admita el reconocimiento del pluralismo jurídico: «es la meta hacia la que se aspira" (p. 509).

Inspirándose de ideas expuestas por Raúl Zaffaroni, Francia afirma que en el artículo 15 del Código Penal se ha previsto el «error culturalmente condicionado". Error que imposibilita la comprensión de la norma y la antijuricidad de la conducta, originado por el acondicionamiento cultural del individuo. En su opinión, es un «error invencible que excluye la culpabilidad y toda sanción penal». Esta interpretación sería la preferible porque, de un lado, es conforme a la concepción expuesta en el artículo comentado, y, de otro lado, «rechaza la asimilación del resto de culturas a la cultura occidental como propuesta política" (p. 511). El autor agrega de manera reiterativa que esta concepción "corresponde con una línea que opta por una sociedad democrática, respetuosa del derecho a la diferencia; es una forma de disminuir el actuar violento del sistema penal y sus efectos negativos» (p. 512).

Hasta aquí lo que dice el autor o, al menos, lo que nosotros creemos honestamente que dice y afirma. 


\section{Comentario}

Siguiendo el mismo esquema escogido por Francia, el primer comentario que se impone concierne el marco ideológico en que ubica el problema tratado. Si la llamada "criminología crítica" ha mostrado, muchas veces y de modo bastante correcto, las deficiencias y paradojas del sistema penal predominante, no ha logrado elaborar un proyecto realista para substituir al sistema criticado. Esto se ha debido a que sus promotores no han estudiado suficientemente la realidad. En nuestro país, hasta donde conocemos, no existe simplemente este tipo de investigaciones. Lo que se hace es repetir los planteamientos teóricos y aplicarlos automáticamente con miras a explicar y enjuiciar el sistema de control penal. Así, se vuelve a cometer el error de siempre: aceptar teorías sin un análisis serio de nuestra realidad.

Francia no ignora este problema. Por lo que se satisface, desde el inicio, a fijar como pautas de su trabajo la afirmación de la «deslegitimación del sistema penal» y de la «necesidad de una alternativa al derecho penal», así como la de "aminorar los efectos negativos del sistema penal» (p. 494). Una discusión objetiva de los problemas relevados resulta imposible porque el autor no explica suficientemente qué quiere decir «deslegitimación del sistema". ¿Cuál es el criterio de referencia para determinar lo que es o no legítimo? ¿Este criterio es la concepción que él atribuye a la criminología crítica o los intereses de los marginados social y culturalmente (urbanos o rurales)? Tampoco explica cuál es la alternativa al derecho penal: ¿un nuevo derecho penal u otro derecho que no recurra a la pena? ¿nuevos derechos penales propios a cada grupo cultural o un derecho penal crítico, democrático para todos? Lo mismo sucede con sus afirmaciones respecto a lo que él llama "colonialismo jurídico", "discurso oficial irreal», "dogmática crítica", "política etnocida", «autonomía cultural».

Este lenguaje genérico puede ser persuasivo por su connotación emotiva, pero no por su fuerza descriptiva de una realidad suficientemente investigada. Esto se pone, particularmente, en evidencia, cuando sugiere que la solución se encuentra en el reconocimiento, primero, del pluralismo jurídico, sin precisar su índole y sus alcances (p. 517); segundo, de los «mecanismos de regulación social y resolución de conflictos no oficiales", sin decir que "hoy cohabitan con nosotros 72 etnias campesinas y nativas" (Jorge Paredes Laos, «El Dominical» de El Comercio, 6.8.95); pero afirmando, al mismo tiempo, que esos mecanismos van "convirtiéndose poco a poco en una forma de reproducción de una nueva cultura y de un poder alternativo y paralelo al oficial» (p. 518), y que tal solución «es la muestra de vitalidad de una cultura que luego de 500 
años de sometimiento se creía en vías de desaparición” (p. 518). Conviene preguntarse: ¿de qué cultura se trata: la andina, la quechua, la aymara, la campa, la aguaruna? ¿y por qué no la negra, la china, la japonesa o la árabe? $O$ en palabras de especialistas: "¿qué puede significar la "revitalización de la cultura indígena", si ya esta cultura no existe como tal, sino profundamente moldeada en torno a nuevos polos de significados, sociabilidad e intercambio?" (Thierry Saignes, "Es posible una historia "chola" del Perú?», en Allpanchis, 35/36, vol. II, p. 650).

Mediante este razonamiento no hacemos sino llevar hasta sus últimas consecuencias las afirmaciones perentoriamente hechas por Francia, quien, sin darse cuenta (pero honestamente), abre la puerta a soluciones peligrosas o poco viables. Por ejemplo, el pluralismo jurídico (reconocimiento de derechos y medios de control a cada grupo cultural) significa el establecimiento de un Estado federativo o la vuelta al sistema de estatutos personales; comportaría un problema de repartición territorial con los riesgos de una balkanización y grandes dificultades prácticas para identificar a los miembros de los grupos culturales debido al mestizaje y a las inmigraciones internas.

A un nivel más concreto se debería aceptar, por ejemplo, que los árabes musulmanes, residentes en nuestro país, practiquen la oblación del clítoris de las niñas por constituir una de sus pautas culturales; que el marido o amancebado, por la misma razón y en ciertos sectores de la sociedad, maltrate a su mujer; que algunas comunidades apliquen castigos corporales a sus miembros cuando así lo establezcan sus costumbres.

Ésta no es, sin duda, la idea de Francia. Así se desprende de su reconocimiento de las deficiencias que tienen los "mecanismos no oficiales [...] ya sea a nivel de derechos humanos o de democratización de su interior". Dos criterios propios a la cultura occidental que parecen ser también utilizados por el autor para determinar, en su lenguaje, la "deslegitimación» del sistema penal oficial y la legitimidad del sistema alternativo (no revelado).

\section{Interpretación del artículo 15 del Código Penal}

Esta manera de analizar, consistente en partir de una concepción determinada (la criminología crítica) para describir una realidad (el sistema penal), sin investigar mayormente los hechos y sin explicar suficientemente las apreciaciones de valor expresadas, es igualmente utilizada por Francia para estudiar el artículo 15 del Código Penal.

En un primer momento, basado en una escasa bibliografía y, sobre todo, foránea, expone lo que él denomina la "teoría general del error". 
Así, pasa revista sucintamente a las nociones de error de tipo y de error de prohibición, comprendiendo en éste el llamado "error de comprensión", introducido, en su opinión, por Raúl Zaffaroni, teniendo en cuenta la «realidad latinoamericana» (p. 503). Siempre según Francia (p. 504), este error afecta la comprensión de la norma penal y la antijuricidad de la conducta, $y$ es un error que se presenta en dos formas: la conciencia disidente ("dificultad en la comprensión por un patrón general de valores distinto al derecho penal oficial, lo que lleva a un deber de conciencia de desobediencia a la ley penal») y el error de comprensión culturalmente condicionado ("la comprensión es imposibilitada por el condicionamiento cultural»). En el primer caso, la culpabilidad es atenuada, y, en el segundo, excluida, porque, según el autor, siempre es un error inevitable (pp. 508, 520).

Sobre esta base teórica, Francia plantea dos posibles interpretaciones del artículo 15. La primera se desprendería de lo afirmado en la exposición de motivos del Código y admitiría que en el artículo 15 sólo estaría prevista la «figura del error de comprensión culturalmente condicionado». Su efecto dependería de la "posibilidad de comprensión": si se halla «imposibilitada se eximirá de pena y cuando solamente se encuentre disminuida se atenuará la pena".

El autor rechaza esta primera interpretación. Primero, porque de admitirla «iríamos en contra de la doctrina que hemos venido siguiendo, la cual unánimemente [!] señala que ante un error de comprensión culturalmente condicionado la pena se excluye al no haber culpabilidad por ser este error siempre un supuesto de error invencible». Se trata de un "argumento de autoridad" porque, de un lado, puede ser resumido diciéndose: "esta interpretación es inaceptable por lo ya dicho por Zaffaronin; de otro lado, la interpretación que descalifica a la primera no ha sido deducida de la disposición legal (art. 15). Su origen está más bien en la afirmación, expresada como dogma: «el habitante de otra cultura no tiene cómo evitar el error y el derecho no puede exigirle dicha comprensión".

La segunda interpretación consiste en comprender que el artículo 15 prevé dos casos: primero, el de la conciencia disidente cuando se refiere a la costumbre; que determina, según Francia, la atenuación de la pena porque el agente del hecho delictuoso obra "sin poder comprender totalmente que dicho acto es un delitom. Segundo, el caso en que el autor "por su cultura no puede comprender [absolutamente, agregamos nosotros] el carácter delictuoso de su acto" (p. 512). Lo determinante sería, entonces, la diferencia existente entre cultura y costumbre. El término cultura indicaría que "el individuo se ha desarrollado con patrones culturales distintos a los del derecho penal oficial y por ello está imposi- 
bilitado para la comprensión de la norma penal». El vocablo costumbre señalaría "un conjunto de patrones y valores que tiene el individuo, los que originan una exigencia de no obediencia a la norma penal» (p. 512).

El argumento decisivo, sin embargo, para tener esta segunda interpretación como la correcta, es el mismo argumento invocado para rechazar la primera. Francia vuelve a repetir (p. 512): por considerarla «acorde con la doctrina seguida y por corresponder con una línea que opta por una sociedad democrática, respetuosa del derecho a la diferencia». Su objetivo ideológico lo manifiesta al final de este párrafo, diciendo que la segunda interpretación "es una forma de disminuir el ámbito de aplicación y ejercicio del sistema penal, así como de disminuir el actuar violento del sistema penal y sus efectos negativos".

\section{Comentario}

Esta sucinta presentación del análisis que hace Francia del artículo 15 del Código Penal muestra su peculiar manera de proceder. Con una finalidad ideológica hace decir a esta disposición legal lo que él considera justo de acuerdo con una concepción del sistema penal, escogida, previamente, como la única democrática, culturalmente diversa e idónea para eliminar los efectos negativos del sistema penal oficial.

En el procedimiento, este razonamiento es muy similar al practicado por los dogmáticos jurídicos tradicionales tan cuestionados por la criminología crítica: un conjunto de principios, teorías o dogmas son considerados como absolutamente verdaderos, y, con su ayuda, se debe explicar, completar o no aplicar disposiciones legales que los contradigan.

Si por interpretación entendemos que es dar un sentido a una disposición legal, el punto de referencia primario e indispensable es el texto legal. El autor del artículo comentado no utiliza debidamente el método literal. Los términos cultura y costumbres no le merecen sino dos breves apreciaciones dogmáticas. Lo mismo hace con las fórmulas «sin poder comprender" o "determinarse de acuerdo a esa comprensión". De haberlo hecho con atención, seguro que se hubiera preocupado por la similitud del artículo 15 con el inciso 1 del artículo 20 del Código Penal. Según este último, el artículo 20, se exime de responsabilidad a quien "por anomalía síquica, grave alteración de la conciencia o por sufrir alteraciones en la percepción, que afectan gravemente su concepto de la realidad, no posea la facultad de comprender el carácter delictuoso de su acto o para determinarse según esta comprensión». En el artículo 15 se establece la misma consecuencia en relación a quien "por su cultura o costumbres 
comete un hecho punible sin poder comprender el carácter delictuoso de su acto o determinarse de acuerdo con esa comprensiónn. De modo que hubiese podido preguntarse si, a pesar de la diferencia lingüística, las dos primeras fórmulas no significan lo mismo. Es decir, si «no poder comprender" no es lo mismo que "no poseer la facultad de comprender». En la misma perspectiva, se podría reflexionar si "poder" y "facultad" no se refieren a la capacidad de poder hacer algo; en el caso concreto, de comprender el carácter delictuoso del acto. Tener en cuenta lo que dicen las demás disposiciones legales para mejor comprender el sentido de la norma que se interpreta, constituye el método sistemático. Su aplicación, relacionando el artículo 15 con el inciso 1 del artículo 20, le hubiera permitido percibir una similitud mayor: ambas disposiciones tienen la misma estructura. La no comprensión del carácter delictuoso del acto o la incapacidad de determinarse son los efectos, en el caso del artículo 15 , de la "cultura" o de las "costumbres" del agente, y, en relación con el artículo 20 , inciso 1 , de una «anomalía síquica», "grave alteración de la conciencia» o "alteración de la percepción». Todas estas circunstancias pueden provocar la incapacidad total de comprender o de determinarse; pero también sólo su disminución parcial (art. 15 in fine, art. 21).

De modo que Francia desnaturaliza totalmente el texto legal cuando establece dos hipótesis diferentes: según la primera, las costumbres determinarían una disminución del poder de comprender, y, de acuerdo con la segunda, la cultura impediría comprender totalmente el carácter delictuoso del acto. Y esto porque de acuerdo con el dogma del que parte, el artículo 15 debe regular el error de comprensión culturalmente condicionado. Así, olvida completamente que el artículo 15 se refiere igualmente al hecho de que el agente no pueda determinarse, total o parcialmente, según la comprensión que tiene del hecho. No ve, de esta manera, que el artículo 15 supone también la posibilidad de que el agente comprenda el carácter prohibido de su acto pero que no sea capaz de determinarse de acuerdo con esta apreciación y esto debido a su "cultura» o a sus "costumbres".

Francia hubiera evitado esta especie de ceguera, debida a la ideología que le esconde la realidad (la disposición legal en vigencia), si hubiera recurrido al método histórico. Es decir, si hubiera indagado sobre los antecedentes legislativos del artículo 15 . Se contenta con recordar la propuesta de Zaffaroni, sin tener la curiosidad de buscar si este autor argentino no había, alguna vez, concretado su idea en algún proyecto o fórmula legislativa. Ahora bien, Zaffaroni ha inspirado claramente la recomendación siguiente: «que se elaboren conceptos doctrinarios que permitan concluir en la inculpabilidad de cualquier persona que, debido a las pautas de su propio grupo cultural, no pueda ajustar su conduc- 
ta a las pautas jurídicas o culturales dominantes» (Zaffaroni, Sistemas penales y derechos humanos en América latina, p. 58).

Pero como toda propuesta es, generalmente, la conclusión de una evolución de ideas, cabe plantearse si la propuesta de Zaffaroni no modificaba y corregía algunas propuestas anteriores. Señalemos, por ejemplo, el proyecto boliviano de 1964, en la medida en que disponía: "el error esencial será también excluyente de culpabilidad, así como el error o la ignorancia de derecho, con particular referencia al indígena", y el proyecto venezolano de 1948 , artículo 13 , que estatuye: «el juez podrá declarar inimputable al indígena que cometa un [...] delito, teniendo en cuenta su incapacidad para comprender o querer, derivada de su inadaptación cultural».

A pesar de la intención de los redactores del artículo 15, expuesta en la exposición de motivos del Proyecto, esta disposición parece afiliarse más al proyecto venezolano, que prevé una causa de inimputabilidad, que al boliviano o a la propuesta de Zaffaroni, que tratan de una circunstancia de inculpabilidad. Esta diferencia posible entre lo que los proyectistas peruanos quisieron prever y el texto que redactaron, permite aceptar que la voluntad del legislador tampoco justifica la interpretación considerada como la verdadera por Francia.

El objetivo que tiene en cuenta Francia para interpretar el artículo 15 puede ser considerado como el mismo que tuvieron los redactores del proyecto: tener en cuenta la pluralidad cultural de nuestra sociedad y así lograr que la reacción punitiva sea practicada atendiendo la especificidad de las pautas de conducta admitidas por el autor del delito. También es aceptable la idea de que el derecho penal debe ser conforme a los principios de democracia, pluralismo cultural y respeto de los derechos humanos.

\section{A manera de conclusión}

Uno de los factores que provoca la ambigüedad del discurso de Francia cuando se refiere al "sistema penal oficial", al "discurso oficial", al "sistema oficial», a la "regulación social y resolución de conflictos oficiales», es el hecho de que no precisa el sentido de estas expresiones. No se sabe con certeza si se refiere al discurso demagógico de los representantes del poder, al sistema real resultante de las constantes violaciones de los principios y disposiciones constitucionales, o al sistema formal estatuido mediante la Constitución. Por esto sólo resulta posible confrontar su propuesta de interpretación del artículo 15 con el marco constitucional oficial, el mismo que casi siempre resulta siendo, sobre todo en países 
como el nuestro, más un proyecto político a realizarse que la regulación de una situación existente.

Otro factor, relacionado con el que viene de ser mencionado, es el esquema maniqueísta en el que encierra su análisis: la alternativa fatal entre "mundo indígena" $y$ "mundo occidental u oficial". Francia mismo trata de salir de esta dicotomía, pero sin llegar a considerar suficientemente los cambios que se han producido y se producen actualmente debidos a la cohabitación e influencias recíprocas (aunque no igualitarias) iniciadas brutalmente con la Conquista. Su defensa de los indígenas, que comprendemos y compartimos, no debe hacer olvidar la complejidad y riqueza del proceso histórico y social que ha dado lugar al Perú actual. Por ejemplo, el pedido de ciudadanía por los caciques y ayllus consistió en exigir tanto sus plenos derechos de fieles "vasallos" del rey de España como su status de plena humanidad. Lo que tenía como consecuencia evitar que un sector de los grupos dominantes encerrase el mundo indígena en su «indianidad», para continuar discriminando y explotando a sus miembros.

Para evitar estos inconvenientes, hubiera sido necesario que Francia planteara mejor el problema del pluralismo cultural y evitara los argumentos demasiado dogmáticos, tanto sobre el sistema de control penal como sobre las nociones de derecho penal que analiza. Esto lo hubiera llevado a discutir la manera como la Constitución regula el multiculturalismo y las jurisdicciones no oficiales. En particular, el artículo 149, que dispone: «las autoridades de las comunidades campesinas y nativas, con el apoyo de las rondas campesinas, pueden ejercer las funciones jurisdiccionales dentro de su ámbito territorial de conformidad con el derecho consuetudinario, siempre que no violen los derechos fundamentales de la persona".

Si hubiera reflexionado sobre este aspecto, seguro que habría cuestionado que se exija, en general, a toda autoridad campesina o nativa, que, al ejercer su jurisdicción conforme al derecho consuetudinario de su comunidad, se plantee la conformidad de éste con los «derechos fundamentales». El conocimiento de éstos supone una relativa integración al sistema oficial. Se habría interrogado si el factor cultural o las costumbres que determinan la incapacidad de comprender o de determinarse, según el artículo 15, deben ser pautas culturales que no contradigan los derechos fundamentales, o si debe considerarse, como lo sugieren los partidarios del relativismo de los derechos humanos, la concepción que cada grupo cultural tiene de los derechos fundamentales.

Las respuestas o explicaciones dadas a estos problemas generales condicionan la solución de los problemas de orden penal y, en especial, la manera de comprender y aplicar los artículos 15 y 20, inciso 1, del Có- 
digo Penal. Dada la naturaleza de esta nota, no nos es posible ir más allá de las explicaciones y sugerencias que hemos hecho al momento de comentar las afirmaciones de Francia. Además, debe tenerse en cuenta que el objetivo principal de esta nota es el de analizar sobre todo la manera como Francia, a pesar de sus conocimientos y seriedad, aborda la interpretación de la ley; mejor dicho, cómo hace dogmática penal.

\section{Bibliografía}

Albo, Xavier (compilador)

1988 Raices de América. El mundo aymara. Madrid.

ARDITO VEGA, Wilfredo

1994 "¿Son los derechos humanos un concepto universal?», en Desfaciendo Entuertos, n. ${ }^{\text {ss }} 3-4$, octubre, p. 31.

Ballón Aguirre, Francisco

1988 Etnia y represión. Lima.

Benitez N., Hernán Darío

1988 Tratamiento juridicopenal del indigena colombiano: ¿inimputabilidad o inculpabilidad? Bogotá.

Cornejo POLAR, Jorge

1991 «El Estado peruano y la cuestión del pluralismo cultural», Cuadernos de Historia, XIII, Universidad de Lima. Facultad de Ciencias Humanas, Lima.

GARCIA SÁNCHEZ, Joaquín

1995 "Amazonía en el proceso de pacificación", en Culturas amazónicas y politica nacional: un nuevo diálogo. CAAAP, Lima, p. 29.

Hurtado Pozo, José

1979 La ley importada. La recepción del derecho penal en el Perú. Lima.

KAPSOLI, Wilfredo

1980 «El pensamiento de la Asociación Pro-indígena». Debates Rurales 3. Cusco.

Llanque Chana, Domingo

1990 La cultura aymara. Destrucción o afirmación de identidad. Lima.

MarTinez, Ana Teresa

1995 «Pluralismo jurídico y diversidad cultural», en Filosofia y sociedad. En busca de un pensamiento crítico. Lima, p. 37. 
Martinon, Jean-Pierre

1987 "Culture (Sociologie de la)", en Ecyclopaedia Universalis, vol. 5, París.

MÉNDEZ, Cecilia

1990 "Los indios, la independencia y la herencia colonial; algunas reflexiones", en Allpanchis, n. ${ }^{\text {os }}$ 35/36, vol. I, p. 139.

Pease G. Y., Franklin

1992 Perú: hombre e historia. Entre el siglo XVI y el XVIII, vol. II. Lima.

Peña Jumpa, Antonio

1994 "Pluralismo jurídico en el Perú», en Desfaciendo Entuertos, n. ${ }^{\text {ss }}$ 3-4, octubre, p. 11.

Pozzi Escot, Inés

1990 "Balance y perspectivas de la educación para poblaciones indígenas en el Perú: 1990", en Allpanchis, n. ${ }^{\text {s }}$ 35/36, vol. II, p. 393.

Rossi, Pietro (editor)

1970 Il concetto di cultura. I fondamenti teorici della scienza antropologica. Traducción de Daniele Pianciola. 2. ${ }^{a}$ ed., Torino.

SAIGNES, Thierry

1990 “Es posible una historia "chola" del Perú?» (Acerca del nacimiento de

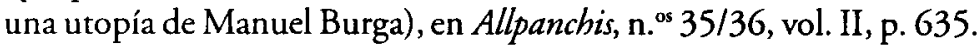

SPALDING, Karen

1974 De indio a campesino. Cambios en la estructura del Perú colonial.

Urteaga Crovetro, Patricia

1994 "Pluralismo legal y semi-autonomía", en Desfaciendo Entuertos, n. ${ }^{\text {os }}$ 3-4, octubre, p. 15.

YRIGOYEN FajARDO, Raquel

1994 "Apuntes sobre el artículo 149 de la Constitución peruana: alcances,

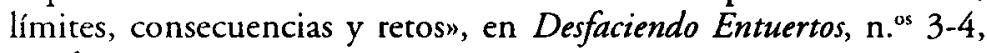
octubre, p. 19.

YRURETA, Gladys

1981 El indigena ante la ley penal. Caracas.

ZAFFARONI, Eugenio R. (coordinador)

1986 «Sistemas penales y derechos humanos en América latina (informe final)». Documento final del programa de investigación desarrollado por el Instituto Interamericano de Derechos Humanos (19821986). Buenos Aires. 